\title{
A RARE ASSOCIATION BETWEEN PRIMARY SJÖGREN'S SYNDROME AND NASOPHARYNGEAL CARCINOMA
}

Andrey Lucas Vieira Rodrigues ${ }^{1, \star}$, Jeniffer Mirelli dos Santos Lopes ${ }^{1}$, Lucas Vasco Aragão ${ }^{1}$, Gabriela de Lira Pessoa Mota ${ }^{1}$, Matheus Henrique Silva Menor ${ }^{1}$, Bárbara Mariana dos Santos Silva ${ }^{1}$, Eduarda Collier de França ${ }^{1}$, Guilherme e Silva Alves ${ }^{1}$, Laísa Nascimento Diniz Teixeira ${ }^{1}$, Maria Laryssa da Silva Pontes ${ }^{1}$, Marina Souto da Cunha Brendel Braga ${ }^{1}$

1.Hospital Getúlio Vargas, Recife (PE), Brazil.

*Corresponding author: andreylucasvr@gmail.com

\section{BACKGROUND}

Sjögren's syndrome (SS) is an inflammatory autoimmune disease that can occur isolated (primary SS) or in association with other autoimmune diseases (secondary SS), like systemic lupus erythematosus or rheumatoid arthritis. The main symptoms are related to the destruction of the exocrine glands, especially the salivary and lacrimal glands, that are reflected by dry mouth and dry eyes. Patients with SS have an elevated risk of developing malignant neoplasms, like non-Hodgkin B-cell lymphoma and other hematologic malignancies. But the association with Nasopharyngeal Carcinoma (NPC) has rarely been reported.

\section{CASE REPORT}

A 37-year-old woman with history of headache in the past two years, unilateral, pulsatile, associated with diplopia, purulent rhinorrhea ipsilateral and appearance of a left cervical mass, compatible with lymphadenopathy, two months before the first evaluation in our service. The patient has a long history of dry eye and dry mouth, without a known diagnostic. Laboratory serologic analysis showed positives anti-RO and anti-LA (239 and $14 \mathrm{U} / \mathrm{mL}$, respectively). Ophthalmologic evaluation confirmed the xerophthalmia by a positive Schirmer test and found a bilateral keratitis. These findings were consistent with the diagnosis of SS. The search for other coexisting autoimmune disease was negative. In the evaluation of the headache with red flags symptoms, the patient was submitted to a brain magnetic resonance imaging (MRI), that showed a bulky expansive process in the nasopharynx, involving the maxillary sinuses bilaterally, posterior ethmoid, skull base, and optic chiasm, with maximum dimensions nearly $7.5 \times 6.5 \mathrm{~cm}$. A nasal endoscopy-guided biopsy of the lesion was performed, with histopathologic result conclusive to undifferentiated nonkeratinizing carcinoma. In accordance with the American Joint Committee on Cancer tumor-node-metastasis staging system, the patient was diagnosed with NPC at stage IV A. She was referred to an oncology service to start chemoradiotherapy.

\section{CONCLUSION}

In patients with SS, we emphasize the possibility of lymphadenopathy in the neck, which might be associated with other cancers than lymphoma, like primary neck and head cancers. The association with NPC is very rare, with only two other reports at the MEDLINE database. 